\title{
MORPHOLOGICAL VARIATIONS OF THE “BABY'S SUPPLY LINE”
}

\author{
C.S. Abaidoo ${ }^{1}$, K.A. Boateng ${ }^{2}$, and M.A. Warren ${ }^{3}$ \\ ${ }^{1}$ Department of Anatomy, School of Medical Sciences, \\ Kwame Nkrumah University of Science sand Technology, Kumasi, Ghana \\ ${ }^{2}$ Department of Pathology, School of Medical Sciences, \\ Kwame Nkrumah University of Science sand Technology, Kumasi, Ghana \\ ${ }^{3}$ Department of Biomedical Science, University of Sheffield, UK
}

\begin{abstract}
To contribute to the sparse information on dimensional variations of umbilical cords in Ghana, 124 placentae with attached umbilical cords were studied. The placentae were obtained from the University of Science and Technology Hospital between 2000 and 2002. Average cord length was 47.04cm (SD 12.8cm) with the shortest cord being $5.5 \mathrm{~cm}$ and the longest $75.50 \mathrm{~cm}$. By empirical definition, $21.56 \%$ of the cords were Short whereas, $78.44 \%$ were long. Most of the umbilical cords (63\%) had empirically eccentric attachments to their placentae; $20.48 \%$ had central and $16.52 \%$ had marginal attachments respectively. Occurrence of furcate insertion of umbilical cord vessels into the placenta was $28 \%$ and non-furcate insertion was $72 \%$. These quantitative data provide baseline values for further investigation.
\end{abstract}

Keywords: umbilical cords, morphology, length, attachments, looping, insertion

\section{INTRODUCTION}

The human umbilical cord is a narrow, complex structure that connects the foetus to the placenta. The umbilical cord is sometimes called the baby's "supply line" because it serves as a conduit for foetal vessels going to and from the mother. Knowledge about the umbilical cord is important because the vessels in the cord are an essential part of the foetal circulation. The umbilical cord has also been found to be a marker of intrauterine complications (Berg and Rayburn, 1995). Morphological variations in the umbilical cord due to its susceptibility to malfor- mations, lesions, mechanical and iatrogenic events throughout pregnancy, labour and delivery, collectively referred to as umbilical cord accidents, are said to be possible causes of foetal injury or death (Harman, 1995). These morphological variations include; umbilical cord attachments to the placenta, extreme umbilical cord lengths, Wharton's jelly content, cord tensile strength, shape, cord looping, cord knotting and umbilical cord vessel morphology and number (Schindler, 1991).

Clinical significance has been imputed to certain variations of umbilical cord and disposition. Cer- 
tain slight variations in the morphological features of umbilical cords have been thought to affect the structure and function of the umbilical cord (Mills et al., 1983; Miller et al., 1982). Length variations in umbilical cord range from total absence to a length of $300 \mathrm{~cm}$ (Stallabrass, 1960; Berg and Rayburn, 1995). A linkage between abnormal cord lengths and neurological abnormalities as well as intelligence quotient (I.Q) values has been reported (Naeye, 1992; Berg and Rayburn, 1995). Umbilical cord abnormalities such as true knots, nuchal coils and body loops have also been suggested to have a close association with extreme umbilical cord lengths, resulting in foetal risks such as foetal malformations, injury and stillbirth (Naeye, 1992). It has been postulated that there may be an increased risk of umbilical cord accidents when a combination of factors such as length of cord, thinness, marginal insertion, nuchal cord and true knot are present in the same foetus (Harman, 1995; Smotherman and Robinson, 1998).

According to Collins (1992) and Flamm (1999) short cords $(<30 \mathrm{~cm})$ could interfere with foetal heart rate patterns and result in an increased need for caesarean section, forceps and vacuum extraction during delivery. In addition, relatively short cord lengths, further compromised by foetal entanglement causes loss of elasticity which could interfere with delivery as well. Umbilical cords that are $70 \mathrm{~cm}$ or longer are considered as long cords (Sornes, 2000) are reported to be associated directly with poor foetal outcome and umbilical cord accidents, especially; foetal entanglement, true knot (sometimes multiple) and torsion (Sornes, 2000). Available literature shows that cords from male foetues tend to have longer lengths than female cords, whilst term vertex foetuses also have longer lengths than term breech foetuses (Sornes, 2000). Additionally multigravida cords might have longer lengths than primagravida cords (i.e. the first pregnancy having a shorter length than the third) (Miller et al., 1982; Sornes and Bakke, 1989).
Twin gestations were also suggested to have foetuses with discordant and shorter lengths than singletons (Sornes, 2000). Umbilical cord function however, does not appear to be impaired by cord length; therefore venous return from the placenta to the foetus was maintained regardless of length (Wright and Ridgway, 1990).

It is estimated that more than 7.6 million perinatal deaths occur world-wide; each year 4.3 million of these are foetal deaths (Schindler, 1991; Sornes, 2000). Ninety-eight percent of perinatal deaths have been said to take place in developing countries, and the perinatal mortality rate is estimated to exceed 55 per 1000 births, which is five times higher than in developed countries (Schindler, 1991). Currently, very little is known about the incidence of foetal deaths resulting from umbilical cord malformations in Ghana. It is therefore considered necessary to establish a comprehensive baseline data on umbilical cord length, cord diameter, cord vessel morphology, cord-placental insertion, and foetal outcome. The distribution of these indices in developing countries is also largely unknown. The occurrence of fetal compression risk based on cord location on the placenta and insertion of umbilical blood vessels is also unknown (Harman, 1995). The present study sought to provide baseline data on the morphological variations of human umbilical cords.

\section{MATERIALS AND METHODS \\ Sample collection}

A total number of one hundred and twenty-four (124) umbilical cords along with placentae were collected from the University of Science and Technology Hospital between 2000-2004. The samples were labelled and preserved in $10 \%$ formalin solution. The umbilical cord segment, from the cut end to the point of insertion on the placenta, was measured with a tape measure (Plate 1) within 30 minutes after fixation.

The measured cord stub attached to the umbilicus of the foetus, which was usually $2.50 \mathrm{~cm}$ was added to the length of the cord attached to the placenta. The position of the cord on the placenta

2 Journal of Science and Technology, Vol. 28, No. 2, August, 2008 


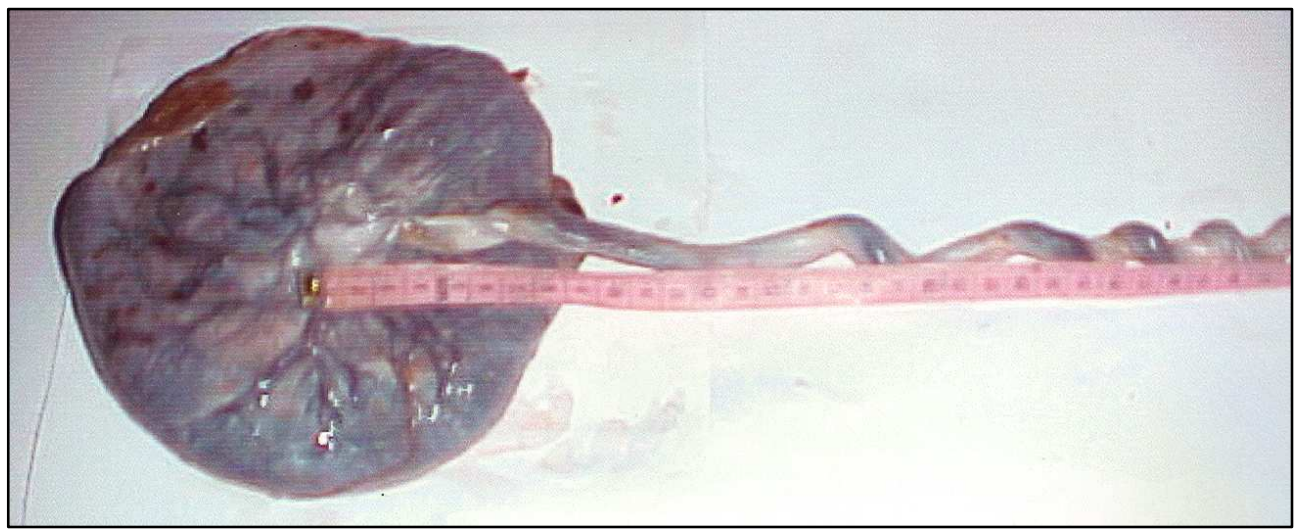

Plate 1: A measuring tape alongside an umbilical attached to its placenta

was also recorded. The insertion point of the cord in relation to the placenta, was located and described as either centric, eccentric or marginal. The insertion of the umbilical vessels into the placentae was examined and described as either furcate (when the vessels were separated from each other before their insertion) or non-furcate (when they were covered by a sheath of Wharton's jelly to their point of insertion). The number of vessels in each umbilical cord was counted and recorded by snipping a bit of each umbilical cord and observing the number of lumina present. The cords were critically examined for knot formation and recorded.

\section{RESULTS}

Umbilical cord length, shape and insertion

The range of cord length obtained was 5.0-75.5 $\mathrm{cm}$ with a mean of $47.04 \mathrm{~cm}$ (SD $12.8 \mathrm{~cm})$. Using the mean cord length in the literature $(\geq 40$ $\mathrm{cm})$ as a marker, the distribution of short and long cords were $21.56 \%$ and $78.44 \%$ respectively. This difference in mean cord length was statistically significant. It was also found that the proportion of long cords was significantly different from that $*$ of short cords using a confidence interval of $95 \%$. A large majority $(69.60 \%)$ of the cords were between $41.50 \mathrm{~cm}$ and $55.00 \mathrm{~cm}$ in length. The diameter of the respective cords was in the range of $1.50 \mathrm{~cm}$ to $3.20 \mathrm{~cm}$ with a mean cord diameter of $2.1 \mathrm{~cm}$ (SD 0.004), using a $95 \%$ confidence interval. A large majority of the cords had a diameter between $2.0 \mathrm{~cm}$ and $2.5 \mathrm{~cm}$. Out of a total number of 124 placentae, central (Plate 2), marginal (Plate 3) and eccentric (Plate 4) insertions constituted $20.48 \%, 16.52 \%$ and $63 \%$ respectively.

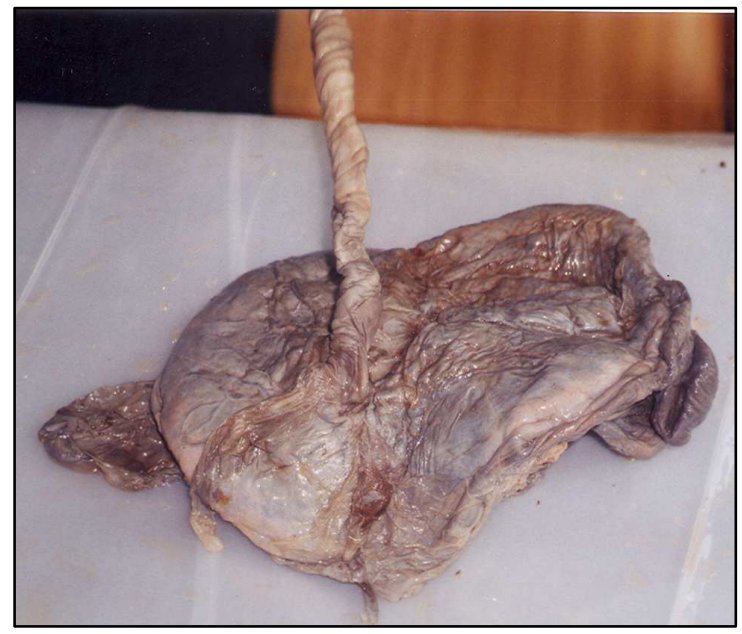

Plate 2: a photograph of an umbilical cord with central attachment to its placenta 


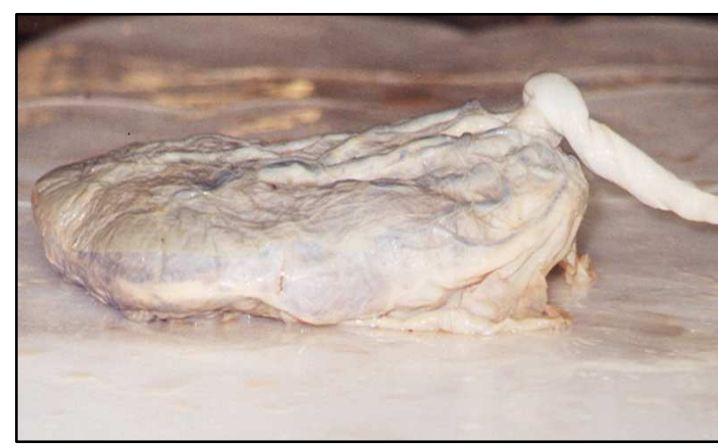

Plate 3: a photograph of an umbilical cord with marginal attachment to its placenta

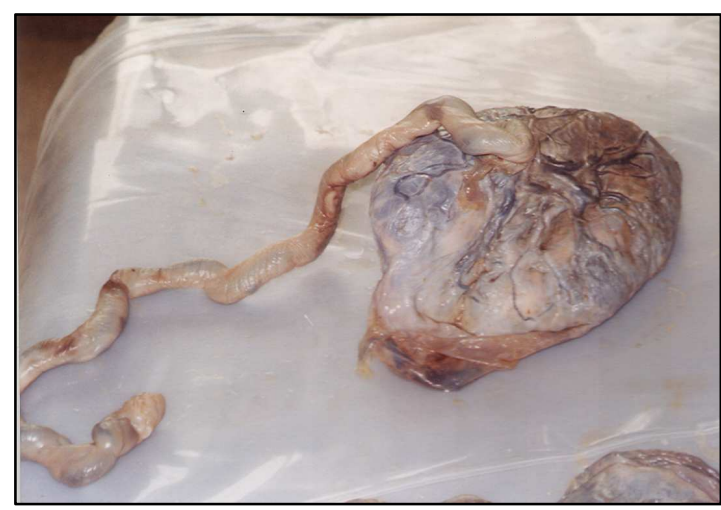

Plate 4: a photograph of an umbilical cord with eccentric attachment to its placenta

\section{Umbilical cord vessels}

The occurrence of 2-, 3- and 4-vessel cords were $1.00 \%, 96.00 \%$ and $2.20 \%$ respectively, indicating that a large majority of the cords had 3 umbilical cord vessels. Out of the 124 umbilical cords studied, one $(0.80 \%)$ had a single umbilical artery. About $8.64 \%$ had furcated attachments (Plate 5) whereas the remaining $91.36 \%$ were non furcated (Plate 5) in their modes of insertion to the placenta. No velamentous umbilical cords were seen.

\section{Cord knots and cord looping}

Knot formation was observed in about five cords. One had a true knot and the remaining four were false knots and the cords in which they were found were considerably long. Looping was quite a prominent feature in the cords. About 50 out of the 124 cords studied presented with cord looping. The occurrence of Hyperhelical (coiled) hypohelical (straight), twisted and spiral cords were $36 \%, 18 \%, 36 \%$ and $10 \%$ respectively.

\section{DISCUSSION}

In the present study cords less than $40.0 \mathrm{~cm}$ were classified as short, those greater than $40.0 \mathrm{~cm}$ were categorized as long. Based on this criterion there were more long cords $(78.44 \%)$ than short

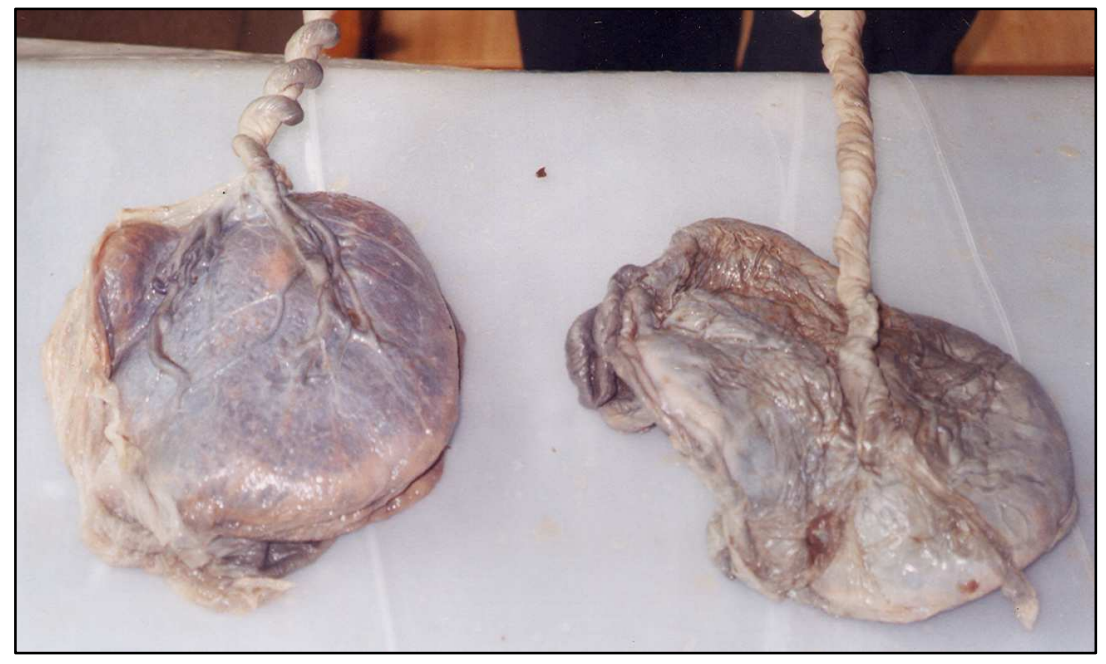

Plate 5: a photograph of two umbilical cords showing furcated insertion (left) and nonfurcated insertion (right)

4 Journal of Science and Technology, Vol. 28, No. 2, August, 2008 
$(21.56 \%)$. This difference in mean cord length was statistically significant. It could be speculated that "normal" cord length should be greater than $40 \mathrm{~cm}$. While opinions differ with regard to the limits of normal cord length, umbilical cord lengths between $40 \mathrm{~cm}$ and $70 \mathrm{~cm}$ have been suggested to be normal (Harman, 1995), whereas short umbilical cords are defined as a total length of less than $40 \mathrm{~cm}$ (Sornes and Bakke, 1989;Harman, 1995). The significance of long umbilical cords resides in the fact that they may be directly associated with poor foetal outcome and umbilical cord accidents such as foetal entanglement, knot formation (multiple) and torsion. Foetal cord entanglements in particular may reduce the volume of blood flow to the foetal brain and limbs and subsequently cause neurological disturbances (Sornes, 2000). Other researchers have reported that, umbilical cord function is not impaired by long cords suggesting that venous return from placenta to foetus is maintained regardless of the length of the umbilical cord (Wright and Ridgway, 1990).

In contrast, relatively short cords may interfere with the mechanics of labour, as the foetus may not be able to descend because the cord length limits it (Hershkovitz et al., 2001). In such cases, Caesarean section, forceps and vacuum extraction would be the only choice left for safe delivery of the foetus. Cords of insufficient length may also result in breech presentation, prolonged labour, abruptio placenta and uterine inversion (Sornes and Bakke, 1989). Short cords are found in newborns with early intrauterine constraint and in those with gross structural or functional limb defects that limit intrauterine movement (Miller et al., 1982).

Umbilical cord length is the only factor associated and documented as a definite risk factor for poor foetal outcome (Hershkovitz et al., 2001). It has been reported that there is an association of abnormal cord length with neurological abnormalities and low IQ values in children who are born with very long umbilical cords (Sornes, 2000).
In the present study it was observed that umbilical cords were commonly positioned eccentrically on placentae (Plate 4). The overall incidence of $83.48 \%$ for a combined centric/eccentric cord insertion is comparable with the observations of Addai et al. (1994). They reported an overall incidence of $74 \%$ for combined centric/eccentric cord insertion and $26 \%$ for marginal/peripheral cords out of 121 sampled umbilical cords. Fox (1978) reported an incidence of $15 \%$ for marginal cords. It is possible that the position and arrangement of marginal insertions may make the cords prone to vessel compression or rupture leading to foetal death (Sornes, 2000). Many researchers have reported that umbilical cords are usually inserted centrally or eccentrically with marginal insertions being rare in occurrence (Salafia, 1990).

It has also been shown that amongst the different types of cord insertions, marginal insertions are generally pose more risk, as a little torsion or stress is likely to cause disruption of blood flow along the cord. It is known that aberrations of umbilical cord attachment can affect the function of the cord. Marginal cords have also been clinically linked with various congenital anomalies (Kamitomo et al., 1999). In the present study it was not possible to do a follow-up on the foetuses with marginal umbilical cords.

A large majority (96\%) of the cords in this study had 3 umbilical cord vessels. Three umbilical cord vessels is normal and adequate for proper fetal development and survival. It is well documented that in humans, the "normal umbilical cord" has an arterial pair that is mildly helical around a straight vein (Cohen et al., 1992; Martinez et al., 1995; Pierce et al., 2001). Therefore differences in umbilical cord vessel morphology may be a risk factor for the foetus and may predispose the foetus to umbilical cord accidents.

Out of the 124 umbilical cords studied, one $(0.80 \%)$ had a single umbilical artery (SUA). Cords with a single umbilical artery, two-vessel cords and four-vessel cords have been reported (Martinez et al., 1995). SUA has been associated 
with stillbirths, with an incidence of $3 \%-20 \%$. Malformations due to inadequate blood supply and brain damage among foetuses with SUA have been reported to be as high as $46 \%$ (Martinez et al., 1995; Schimmel and Eidelman, 1998). In a report Sornes, (2000) it was found that about $27 \%$ of livebirths with structural anomalies were associated with SUA while in specimens obtained from early abortions, foetal deaths and autopsies, SUA was seen in $66.3 \%$. Four-vessel-cords have also been associated with foetal abnormalities (Martinez et al., 1995; Schimmel and Eidelman, 1998). Cases of five or more cords, are the numerous variations associated with conjoined twining (Martinez et al., 1995).

The incidence of umbilical vessel attachments was found to be $8.64 \%$ and $91.36 \%$ for furcate and non-furcate respectively. The occurrence of non-furcate vessels was significantly higher than furcate ones probably suggesting that nonfurcate vessels are normal vessel attachments whereas furcate vessels could result in injury and haemorrhage due to lack of adequate protection for these vessels. Pierce et al. (2001) reported that the mode of umbilical cord insertion has no significant effect on the examined components, and such differences are probably the effect of biological variations during normal placental and fetal development. However, more recently it has been reported that the occurrence of foetal compression risk based on cord insertion and location is still unknown (Sornes, 2000).

The cord diameter in the present study was found to be between $2.00 \mathrm{~cm}$ to $2.50 \mathrm{~cm}$ this however compares quite favorably with the findings of Sornes, (2000) who described the typical umbilical cord as having a fairly uniform diameter of $2.0-2.5 \mathrm{~cm}$. In the present study the minimum cord diameter was about $1.5 \mathrm{~cm}$. Cord diameter may be related to its Wharton's jelly content. It may be that umbilical cords of larger diameter have a large amount of Wharton,s jelly whereas cords with smaller diameters have rela- tively little jelly content. Wharton's jelly has a gelatinous consistency and may serve as a cushion for the umbilical vessels. Therefore lean umbilical cords may also be more vulnerable to compression and breaking due to lack of tensile strength. However in a recent study by AmielTison and Stewart (1994), it was reported that cords with little or inadequate Wharton's jelly may suggest poor nutrition and lack of glycogen in fetal tissues.

The occurrence of Hyperhelical (coiled) hypohelical (straight), twisted and spiral cords were $36 \%, 18 \%, 36 \%$ and $10 \%$ respectively. Out of 124 umbilical cords studied, one had knot formation and another had constrictions. The coiled and twisted features found in cords could be as a result of tension, stress or strain resulting from the weight of the foetus which is borne normally by the elastic nature of the umbilical cord. However excessive pressure on cords could compromise the elasticity of cords leading to the occurrence of straight cords. In addition, excessive coiling and twisting could however lead to the interference in blood flow through the vessels.

Knot formation was seen in about five umbilical cords. Although four of these knots were false, the cords in which they were found were considerably long in length $(65-75.5 \mathrm{~cm})$. False cord knots are quite frequent in normal pregnancies, and appear to be due to umbilical artery loops within the cord (Sornes, 2000; Hershkovitz et al., 2001). Knotting in the umbilical cord is a sequence of events which creates a loop of cord counter to the twist (torque) applied by foetal movements (which may be due to blood flow disturbances). This loop passes over the foetal body with time and occasionally, a bunch of cord loops make the false impression of a cord knot.

False umbilical cord knots may have little clinical significance whereas true knots of the umbilical cord although rare may lead to obstruction of the fetal circulation and subsequent intrauterine death (Hershkovitz et al., 2001). In addition, evidence of fetal heart rate changes, umbilical blood flow

6 Journal of Science and Technology, Vol. 28, No. 2, August, 2008 
reduction, decreased fetal movement has been reported with cord knotting (Sornes, 2000).

Looping was quite a prominent feature in the cords. Out of the 124 umbilical cords, about 91 presented with cord looping. This could be compared to the loops in the cord of a telephone receiver. Looping is an adaptative anatomical feature, used by the human body to contain long objects in a small space (Lacro et al., 1987). Cords with relatively wide diameters exhibited less looping than cords with smaller diameters. This might be a way for the smaller cords to make up for the inadequate tensile strength and adapt to torsion. It has been suggested by Strong et al. (1993) that non-coiled cords and poorly coiled cords are structurally less able to resist external compressive forces which may lead to preterm delivery, operative delivery for fetal distress and intrauterine death.

The coiled and twisted features found in cords could be as a result of tension, stress or strain resulting from the weight of the foetus which is borne normally by the elastic nature of the umbilical cord. However excessive pressure on cords could compromise the elasticity of cords leading to the occurrence of straight cords. In addition, excessive coiling and twisting could however lead to the interference in blood flow through the vessels.

Ben-Arie et al. (1995) reported that Hyperhelical (coiled) hypohelical (straight), true knot and constriction were $1 \%, 5 \%, 1-25 \%$ and $1 \%$ respectively. Utsu and Maeda (1991) and Glanfield and Watson (1986) stated that stillbirth was associated with torsion. Randall (1989) however proposed that umbilical cords under the influence of torsion could be untwisted, with Vandeplassche et al. (1986) confirming that torsioncompromised umbilical cords were different from natural helixes which cannot be untwisted. These torsion twists, if not resolved as early as possible could cause blood flow obstruction through the umbilical cord when the ability of the cord to absorb the torque is exceeded (Lacro et al., 1987). In another study, Collins and Collins (2000) indicated that tension, if applied to umbilical cords could lead to kinking which invariably blocks blood flow.

\section{CONCLUSION}

In the present study the overall incidence of $85.18 \%$ for a combined centric/eccentric cord insertion suggests that umbilical cords studied were commonly positioned eccentrically or centrally on placentae and the vessels were inserted in a non-furcate fashion. Results of the present study provide for the first time detailed baseline data on the variations in the morphological characteristics of the umbilical cord such as length, type of insertion, number of vessels and type of vessel attachment.

\section{REFERENCES}

Addai, F.K., Quarshie, F.J.K., Ockleford, C. D. (1994). The mode of insertion of umbilical cord and vessels :associatin with maternal haemoglobin genotype, neonatal factors and placental component volumes. Anatomy and Embryology. 189 :107-114.

Amiel-Tison, C., Stewart, A. (1994). The newborn infant; one brain for life. INSERM; Paris, France.

Ben-Arie, A., Wessman, A., Steinberg, Y., Levy, R., Hagay, Z. (1995). Oligohydramnios, intrauterine growth retardation and foetal death due to umbilical cord torsion. Archaeology of Gynecology and Obstetrics. 256:159-161.

Berg, T.G., Rayburn, W.F. (1995). Umbilical Cord Length and Acid-Base Balance at Delivery. Journal of Reproductive Medicine. 40:9-12.

Cohen, H.L., Shapiro, M. L., Haller, J.O., Schwartz, D. (1992). The Multivessel Umbilical Cord: An Antenatal Indicator of Possible Conjoined Twinning. Journal of Clinical Ultrasound. 20:278-282.

Collins, J. H. (1992). The short umbilical cord. 
American Journal of Obstetrics and Gynecology. 166:268-69.

Collins, J.H., Collins, C.L. (2000). The Human Umbilical Cord: United Kingdom Journal, Jauniaux E, O'Brien S. (editions): The Placenta: Basic Science and Clinical Practice. London, RCOG Press, Ch.26 pp.319-329.

Flamm, B.L. (1999). Tight nuchal cord and shoulder dystocia: A potentially catstrophic combination. American Journal of Obstetrics and Gynecology. 94:853.

Fox, H. (1978). Pathology of the umbilical cord. In: pathology $f$ the placenta. Saunders, London. Pages 426-457.

Glanfield, P.A., Watson, R. (1986). Intrauterine foetal death due to torsion. Arch. Pathology of Laboratory Medicine. 110:357-358.

Hanson, M., Kiserud, T. (2001). Cardiovascular System in Fetal growth and development. Harding R, Bocking AD editions. Cambridge University Press pg. 70-93.

Harman, C.R. (1995). Invasive Techniques in the management of Allimmune Anaemia. In: Harman C. R. Invasive Foetal Testing and Treatment: Cambridge MA, Blackwell Scientific Publishers, p. 109-191.

Hershkovitz, R., Sisberstein, T., Sheiner, E., Shoham-Vardi, I., Holcberg, G., Katz, M. (2001). Risk factors associated with true knots of the umbilical cord. European Journal of Obstetrical and Gynecological Reproductive Biology 98:36-39.

Itskovitz, J., LaGamma, E.F., Rudolph, A.M. (1987). Effects of cord compression of foetal blood flow distribution and delivery. American Journal of Physiology. 252:H100H109.

Jones, A. (1998). Truly knotted cords. Australian and New Zealand Journal of obstetrics and Gynecology. 381:98-99.

Kamitomo, M., Sueyoshi, K., Matsukita, S., Matsuda, Y., Hatae, M., Ikenoue, T. (1999).
Hemangioma of the umbilical cord: stenotic change of the umbilical vessels. Foetal Diagnostic Therapy 14:328-31.

Lacro, R.V.,Jones, K.L., Bennirschke, K. (1987). The umbilical cord twist: Origin, direction and relevance. American Journal of Obstetrics and Gynecology. 157;833-838.

Malpas, P., Symonds, E. M. (1966). Observations of the structure of the human umbilical cord. Surgical and Gynecological Obstetrics. 123: page 750 .

Martinez, J.M., Comas, C., Antolin, E., Borrell, A., Puerto, B., Casals, E., Mallofre, M.C., Furtuny, A. (1995). Biochemical and Doppler predictors of poor perinatal outcome in a foetus with four umbilical vessels. European Journal of Obstetrical and Gynecological Reproductive Biology. 62:145-147.

Miller, M.E., Jones, M.C. Smith, D.W. (1982). Tension: The basis of umbilical cord growth. Journal of Paediatrics 101:844.

Mills, J. L., Harley, E. E., Moessinger, A. C. (1983). Standards for measuring umbilical cord length. Placenta 4:423-426.

Naeye, R.L. (1992). Disorders of the umbilical cord. In Disorders of the Placenta, Foetus and Neonate: Diagonosis and Clinical Significance. Mosby Year Book St. Louis, MO:92-117.

Pierce, B.T., Dance, V.D., Wagner, R.K., Apodaca, C.C., Nielsen, P.E., Calhoun, B.C. (2001). Perinatal outcome following foetal single umbilical artery diagnosis. Journal of Maternal and Foetal Medicine 10:59-63.

Randall, G.C.B. (1989). Form and development of the umbilical cord in pigs and their association with the delivery of viable pigs. American Journal of Veterinary Research. 50:1512-1515.

Salafia, C. (1990). Why all placenta should be examined by a pathologist in American Journal of Obstetrics and Gynaecology 163:128293. 
Schimmel, M.S. E idelman, A. I. (1988). Supernumery umbilical vein rsulting in fourvessel umbilical cord. American Journal of Perinatology. 15:299-301.

Schindler, N.R. (1991). Importance of the placenta and cord in the defense of neurologically impaired infant claims. Archeological Pathology of Laboratory Medicine. Editions 7:685-687.

Smotherman, W.P., Robinson, S. R. (1988). Behaviour of the Foetus. Caldwell, New Journal. The Telford Press.

Sornes T., (2000). Umbilical Cord Knots. Acta Obstetricia et Gynecologica Scandinavica 79:157-159.

Sornes, T., Bakke, T. (1989). Uterine size, Parity and Umbilical Cord length. Acta Obstetrics and Gynecologyof Scandinavia 68:43941.

Stallabrass, P. (1960). Long Cord. British Medical Journal. August, 603.
Strong, T. H., Elliot, J. P., Radin, T. (1993). Noncoiled umbilical blood vessels: a new marker for the fetus at risk. Obstetrics and Gynecology;81:409-11.

Utsu, M., Maeda, K. (1991). Distinction of the pathologic hyper-torsion from the physiological spiral development of the umbilical cord. Choopna igaku Japanese Journal of Medical Ultrasonics. 18:607-608.

Vandeplassche, M., Lauwers, H. (1986). The Twisted Umbilical Cord: An expression of kinesis of the equine foetus. Animal Reproduction Science 10:163-175.

Wright, J W., R Ridgway, L.E. (1990). Sources of variability in the umbilical artery systolic/ diastolic ratios: Implications of the Poiseuille equation. American Journal of Obstetrics and Gynecology 163: 1788-91.

Zhou, C. J. (1990). Foetal distress caused by umbilical cord anomalies; analysis of 456 cases. Journal of Practical Obstetrics and Gynecology. 6:316-17. 